\title{
Nodal Domains in Chaotic Microwave Rough Billiards with and without Ray-Splitting Properties
}

\author{
O. Hul ${ }^{a}$, N. SavytskyY ${ }^{b}$, O. TymoshchuK ${ }^{a}$, S. Bauch $^{a}$ \\ AND L. SIRKO ${ }^{a}$ \\ ${ }^{a}$ Institute of Physics, Polish Academy of Sciences \\ al. Lotników 32/46, 02-668 Warszawa, Poland \\ ${ }^{b}$ R\&D Vector Sp. z o.o., Krzemowa 6, 81-577 Gdynia, Poland
}

\begin{abstract}
We study experimentally nodal domains of wave functions (electric field distributions) lying in the regime of Breit-Wigner ergodicity in the chaotic microwave half-circular ray-splitting rough billiard. Using the rough billiard without ray-splitting properties we also study the wave functions lying in the regime of Shnirelman ergodicity. The wave functions $\Psi_{N}$ of the ray-splitting billiard were measured up to the level number $N=204$. In the case of the rough billiard without ray-splitting properties, the wave functions were measured up to $N=435$. We show that in the regime of Breit-Wigner ergodicity most of wave functions are delocalized in the $n, l$ basis. In the regime of Shnirelman ergodicity wave functions are homogeneously distributed over the whole energy surface. For such wave functions, lying both in the regimes of Breit-Wigner and Shnirelman ergodicity, the dependence of the number of nodal domains $\aleph_{N}$ on the level number $N$ was found. We show that in the regimes of Breit-Wigner and Shnirelman ergodicity least squares fits of the experimental data reveal the numbers of nodal domains that in the asymptotic limit $N \rightarrow \infty$ coincide within the error limits with the theoretical prediction $\aleph_{N} / N \simeq 0.062$. Finally, we demonstrate that the signed area distribution $\Sigma_{A}$ can be used as a useful criterion of quantum chaos.
\end{abstract}

PACS numbers: 05.45.Mt, 05.45.Df

\section{Introduction}

Distribution of nodal domains of real wave functions $\Psi(x, y)$ in 2D quantum systems (billiards) have been considered in recent theoretical papers by Blum et al. [1] and Bogomolny and Schmit [2]. Nodal lines $\Psi(x, y)=0$ divide a wave 
function $\Psi(x, y)$ into nodal domains, regions with definite signs. Blum et al. [1] have shown that the distributions of nodal domains are different for systems with integrable and chaotic underlying classical dynamics. Thus a new criterion, not directly related to spectral statistics, of quantum chaos was provided. Bogomolny and Schmit [2] have proposed a percolation-like model to describe properties of the nodal domains of generic chaotic system and have shown that the distribution of nodal domains of such systems is universal.

The first experimental investigation of nodal domains of wave functions lying in the regime of Shnirelman ergodicity in the chaotic microwave rough billiard without ray-splitting has been reported by Savytskyy et al. [3].

In this paper we present the experimental investigations of nodal domains of wave functions of the chaotic microwave rough billiards without and with ray-splitting phenomena. Ray-splitting systems are a new class of chaotic systems in which the underlying classical mechanics is non-deterministic and non-Newtonian [4-7]. In ray-splitting systems waves which encounter a discontinuity in the propagation medium split into two or more rays travelling usually away from the discontinuity. Ray-splitting occurs in many fields of physics, whenever the wave length is large in comparison with the range over which the potential changes. A well-known example of ray-splitting in optics is reflection and refraction of light entering a dielectric medium. A very useful model systems for the investigation of ray-splitting phenomena are microwave cavities with dielectric inserts [5, 8-10].

Throughout this paper we use the following name convention: billiards with ray-splitting are called RS billiards, billiards without ray-splitting properties are called no-RS billiards.

In the case of RS rough billiard we focus our attention on the regime of Breit-Wigner ergodicity. However, for no-RS rough billiard we also consider the regime of Shnirelman ergodicity.

In both cases the dependence of the number of nodal domains $\aleph_{N}$ on the level number $N$ was found. For this purpose we used a new method of the reconstruction of wave functions introduced by Savytskyy and Sirko [11]. In the case of the half-circular microwave RS rough billiard and no-RS rough billiard this method allowed for the reconstruction of wave functions with the level numbers $N \leq 204$ and $N \leq 435$, respectively.

\section{Experiment}

In the experiment we use thin (height $h=8 \mathrm{~mm}$ ), rough, made of aluminium half-circular microwave cavities (Fig. 1), which due to the equivalence between the Schrödinger equation and the Helmholtz equation [7, 12] simulate quantum billiards. The rough RS billiard is simulated by the microwave RS cavity with the dielectric (Teflon) insert of radius $R_{d}=8.465 \mathrm{~cm}$ (see Fig. 1). The cutoff frequencies are $\nu_{\mathrm{c}}=c / 2 \eta h \simeq 13.1 \mathrm{GHz}$ and $\nu_{\mathrm{c}}=c / 2 h \simeq 18.7 \mathrm{GHz}$ for the RS and no-RS billiards, respectively. $c$ is the speed of light and $\eta=1.425$ 
is the index of refraction of the Teflon insert. The cavity sidewalls consist of 2 segments. The rough segment 1 is described by the radius function $R(\theta)=$ $R_{0}+\sum_{m=2}^{M} a_{m} \sin \left(m \theta+\phi_{m}\right)$, where for the RS rough billiard the mean radius $R_{0}=20.0 \mathrm{~cm}, M=20, a_{m}$ and $\phi_{m}$ are uniformly distributed on [0.084, 0.091] $\mathrm{cm}$ and $[0,2 \pi]$, respectively, and $0 \leq \theta<\pi$. In the case of the no-RS billiard the mean radius $R_{0}=20.0 \mathrm{~cm}, M=20, a_{m}$ and $\phi_{m}$ are uniformly distributed on $[0.269,0.297] \mathrm{cm}$ and $[0,2 \pi]$, respectively, with $0 \leq \theta<\pi$. The half-circular geometry of the cavities is very suitable because it allows one to exclude nearly degenerate low-level eigenstates $[13,14]$. It also simplifies the measurements of the electric field distribution inside the billiard.
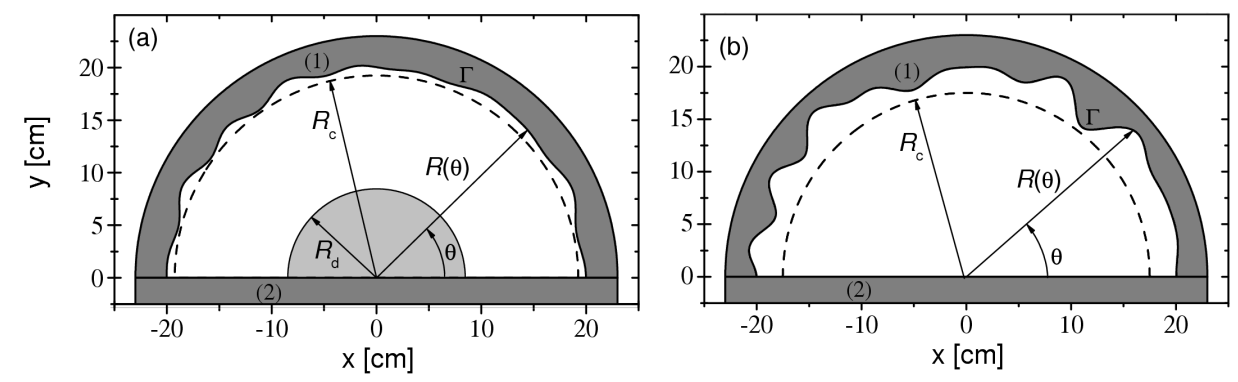

Fig. 1. (a) Sketch of the chaotic half-circular microwave ray-splitting rough billiard in the $x y$ plane. Dimensions are given in $\mathrm{cm}$. The cavity sidewalls are marked by 1 and 2 (see the text). Squared wave functions $\left|\Psi_{N}\left(R_{\mathrm{c}}, \theta\right)\right|^{2}$ were evaluated on a half-circle of fixed radius $R_{\mathrm{c}}=19.25 \mathrm{~cm}$. The dielectric Teflon insert of radius $R_{d}=8.465 \mathrm{~cm}$ was placed inside the cavity. Billiard's rough boundary $\Gamma$ is marked with the bold line. (b) The chaotic half-circular microwave no-RS rough billiard in the $x y$ plane. Squared wave functions $\left|\Psi_{N}\left(R_{\mathrm{c}}, \theta\right)\right|^{2}$ were evaluated on a half-circle of fixed radius $R_{\mathrm{c}}=17.5 \mathrm{~cm}$.

The roughness of a billiard, which may be characterized by the function $k(\theta)=(\mathrm{d} R / \mathrm{d} \theta) / R_{0}[15]$, determines its dynamics and other properties. The roughness parameters $\tilde{k}$ defined as the angle average of the function $k(\theta)$ were for the RS and no-RS billiards $\tilde{k}=\left(\left\langle k^{2}(\theta)\right\rangle_{\theta}\right)^{1 / 2} \simeq 0.200$ and 0.488 , respectively. In such billiards the dynamics is diffusive in orbital momentum due to collisions with the rough boundary because the roughness parameter $\tilde{k}$ is much larger than the chaos border parameter $k_{\mathrm{c}}=M^{-5 / 2}=0.00056$ [15]. The eigenstates of a billiard are localized [16] for the level number $N<N_{\mathrm{e}}=1 / 128 \tilde{k}^{4}$. The border of BreitWigner regime is given by $N_{\mathrm{W}}=M^{2} / 48 \tilde{k}^{2}$. It means that between $N_{\mathrm{e}}<N<N_{\mathrm{W}}$ Breit-Wigner ergodicity [17] ought to be observed and for $N>N_{\mathrm{W}}$ Shnirelman ergodicity should emerge. In the regime of Shnirelman ergodicity wave functions have to be uniformly spread out in the billiard [18]. The borders in the billiards under consideration are the following: for the RS billiard $N_{\mathrm{e}}=5$ and $N_{\mathrm{W}}=208$; for the no-RS billiard $N_{e}=1$ and $N_{W}=35$. 
We determine wave functions using a method described in [11], which is based on the measurements of the intensity of the electric field $\left|E_{N}\left(R_{\mathrm{c}}, \theta\right)\right|^{2}$ on a half-circle of a fixed radius $R_{\mathrm{c}}$ (see Fig. 1) by the perturbation technique and construction of "trial functions".

In the perturbation technique [19-22] a small perturber is introduced inside the cavity to alter its resonant frequency according to

$$
\nu-\nu_{N}=\nu_{N}\left(a B_{N}^{2}-b E_{N}^{2}\right)
$$

where $\nu_{N}$ is the $N$ th resonant frequency of the unperturbed cavity, $a$ and $b$ are geometrical factors. Equation (1) can be used to evaluate the square of electric field $E_{N}$ only when the term containing magnetic field $B_{N}$ may be neglected. A small perturber, a piece of metallic pin $(3.0 \mathrm{~mm}$ in length and $0.25 \mathrm{~mm}$ in diameter $)$ was used in order to minimize the influence of $B_{N}$ on the frequency shift $\nu-\nu_{N}$. The perturber was attached to the line hidden in the groove $(0.4 \mathrm{~mm}$ wide, $1.0 \mathrm{~mm}$ deep) made in the cavity's bottom wall along the half-circle of radius $R_{\mathrm{c}}$ and moved by the stepper motor. The application of such a small pin perturber limited the largest positive frequency shifts to the uncertainty of frequency shift measurements $(15 \mathrm{kHz})$. It was found that the presence of the narrow groove in the bottom wall of the cavity caused only very small changes $\delta \nu_{N}$ of the eigenfrequencies $\nu_{N}$ of the cavity $\left|\delta \nu_{N}\right| / \nu_{N} \leq 10^{-4}$. It means that its influence on the structure of the cavity's wave functions was negligible. The influence of the thermal expansion of the aluminium and Teflon insert on eigenfrequncies of cavities was eliminated by stabilizing the temperature of the cavity with the accuracy of $0.05^{\circ}$.

The experimental set-up consisting of a microwave cavity, a microwave synthesizer HP 8672A, a lock-in amplifier SR530, a stepper motor for precise moving of a line with the attached perturber, an antenna with a coupler and a crystal detector, and a computer is shown in Fig. 2.

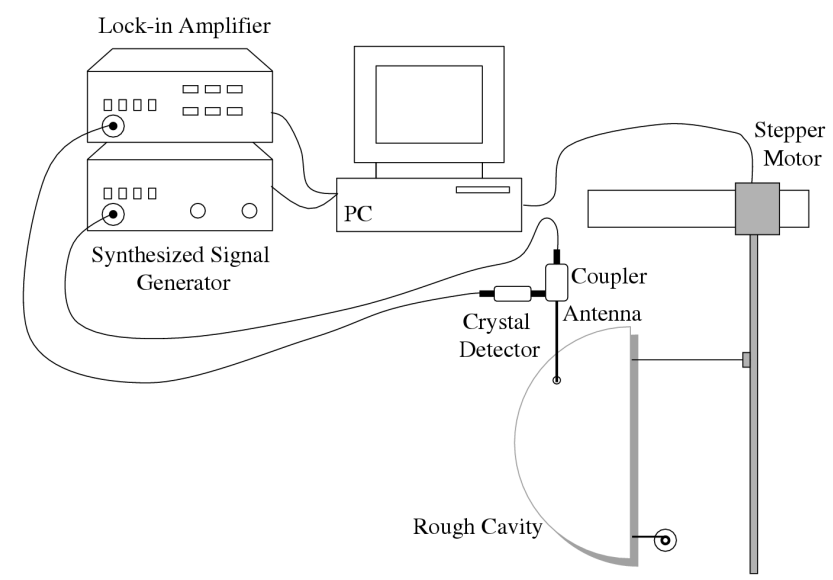

Fig. 2. The experimental set-up for measurements of wave functions (electric field distributions) in the microwave rough billiards by the means of the perturbation technique. 
For the RS and no-RS cavities the measurements were performed at $0.36 \mathrm{~mm}$ steps along a half-circles with fixed radius $R_{\mathrm{c}}=19.25 \mathrm{~cm}$ (1674 steps) and $R_{\mathrm{c}}=$ $17.5 \mathrm{~cm}$ (1531 steps), respectively. This step was small enough to reveal in details the space structure of high-lying wave functions. For a given position of the perturber the resonance frequencies were measured with the step of $50 \mathrm{kHz}$ for lower, narrower resonances, and of $100 \mathrm{kHz}$ for the higher and wider ones. The measurements were time consuming and depending on the level number $N$ lasted from 2 hours for the lower levels to 4 hours for the higher ones.

The perturbation technique of measurements allows for the extraction of information about the wave function amplitude $\left|\Psi_{N}\left(R_{\mathrm{c}}, \theta\right)\right|$ at any given point of the cavity but it does not allow one to determine the sign of $\Psi_{N}\left(R_{\mathrm{c}}, \theta\right)$ [23]. The knowledge of the sign of the wave function $\Psi_{N}\left(R_{\mathrm{c}}, \theta\right)$ is necessary in the procedure of the reconstruction of the wave function $\Psi_{N}(r, \theta)$ of the billiard. In the papers $[3,11]$ the following effective sign-assignment procedure was presented which bases on the identification of all close to zero minima of $\left|\Psi_{N}\left(R_{\mathrm{c}}, \theta\right)\right|$. In this procedure the sign "minus" is arbitrarily assigned to the region between the first and the second minimum. The sign "plus" is assigned to the region between the second minimum and the third one and so on. As a result, the "trial wave function" $\Psi_{N}\left(R_{\mathrm{c}}, \theta\right)$ is constructed. If the assignment of the signs is correct the wave function $\Psi_{N}(r, \theta)$ of the billiard should be reconstructed with the boundary condition $\Psi_{N}\left(r_{\Gamma}, \theta_{\Gamma}\right) \simeq 0$.

\section{Reconstruction of wave functions}

In the procedure of the reconstruction of wave functions of a rough RS half-circular microwave billiard one should take into account the fact that inside the billiard exist two different regions. The wave function outside of the half-circular Teflon insert $\left(r \geq R_{\mathrm{d}}\right)$ may be expanded in terms of Hankel functions

$$
\Psi_{N}^{\text {out }}(r, \theta)=\sum_{s=1}^{L} a_{s} \Omega_{s}\left(k_{N} r\right) \sin (s \theta)
$$

where $\Omega_{s}(x)=\operatorname{Re}\left(H_{s}^{(2)}(x)+S_{s s}\left(k_{N} R_{\mathrm{d}}\right) H_{s}^{(1)}(x)\right)$ and $k_{N}=2 \pi \nu_{N} / c . H_{s}^{(1)}(x)$ and $H_{s}^{(2)}(x)$ are Hankel functions of the first and the second kind, respectively. The matrix $S_{s s^{\prime}}\left(k_{N} R_{\mathrm{d}}\right)$ is defined as follows [24]

$$
S_{s s^{\prime}}\left(k_{N} R_{\mathrm{d}}\right)=-\frac{H_{s}^{(2)^{\prime}}\left(k_{N} R_{\mathrm{d}}\right)-\eta\left[J_{s}^{\prime}\left(\eta k_{N} R_{\mathrm{d}}\right) / J_{s}\left(\eta k_{N} R_{\mathrm{d}}\right)\right] H_{s}^{(2)}\left(k_{N} R_{\mathrm{d}}\right)}{H_{s}^{(1)^{\prime}}\left(k_{N} R_{\mathrm{d}}\right)-\eta\left[J_{s}^{\prime}\left(\eta k_{N} R_{\mathrm{d}}\right) / J_{s}\left(\eta k_{N} R_{\mathrm{d}}\right)\right] H_{s}^{(1)}\left(k_{N} R_{\mathrm{d}}\right)} \delta_{s s^{\prime}}
$$

The derivatives of Hankel and Bessel functions are marked by primes. In Eq. (2) the number of basis functions is limited to $L=k_{N} r_{\max }^{\mathrm{RS}}+4$, where $r_{\max }^{\mathrm{RS}}=20.7 \mathrm{~cm}$ is the maximum radius of the RS cavity. $k_{N} r_{\max }^{\mathrm{RS}}$ is a semiclassical estimate for the maximum possible angular momentum for a given $k_{N}$. The functions with angular momentum $s>k_{N} r_{\max }^{\mathrm{RS}}$ describe evanescent waves. We checked that the basis of $L$ wave functions was large enough to properly reconstruct billiard's wave 
functions. The coefficients $a_{s}$ in Eq. (2) may be determined from the "trial wave functions" $\Psi_{N}\left(R_{\mathrm{c}}, \theta\right)$

$$
a_{s}=\left[\frac{\pi}{2} \Omega_{s}\left(k_{N} R_{\mathrm{c}}\right)\right]^{-1} \int_{0}^{\pi} \Psi_{N}\left(R_{\mathrm{c}}, \theta\right) \sin (s \theta) d \theta .
$$

Inside the Teflon insert $\left(r \leq R_{\mathrm{d}}\right)$ the wave functions of the RS billiard may be expanded in terms of circular waves

$$
\Psi_{N}^{\mathrm{in}}(r, \theta)=\sum_{s=1}^{L^{\prime}} a_{s}^{\prime} J_{s}\left(\eta k_{N} r\right) \sin (s \theta)
$$

The number of basis functions is limited to $L^{\prime}=\eta k_{N} R_{\mathrm{d}}$. The coefficients $a_{s}^{\prime}$ in Eq. (5) can be evaluated from the continuity condition fulfilled at the border of the dielectric insert $\Psi_{N}^{\text {out }}\left(R_{\mathrm{d}}, \theta\right)=\Psi_{\mathrm{N}}^{\text {in }}\left(\mathrm{R}_{\mathrm{d}}, \theta\right)$ allowing in this way to reconstruct the full wave function $\Psi_{N}(r, \theta)$ of the billiard.

The refraction index $\eta=1.425 \pm 0.002$ of Teflon was found experimentally by measuring the set of resonant frequencies of a microwave circular cavity of radius $R_{T}=3.25 \mathrm{~cm}$ entirely filled by it.

The procedure of determining of wave functions of a no-RS half-circular billiard is much simpler. Inside the whole interior of the billiard the wave functions may be expanded in terms of circular waves (here only odd states in expansion are considered)

$$
\Psi_{N}(r, \theta)=\sum_{s=1}^{L^{\prime \prime}} a_{s}^{\prime \prime} J_{s}\left(k_{N} r\right) \sin (s \theta)
$$

where the coefficients $a_{s}^{\prime \prime}$ may be extracted from the "trial wave function" $\Psi_{N}\left(R_{\mathrm{c}}, \theta\right)$ via

$$
a_{s}^{\prime \prime}=\left[\frac{\pi}{2} J_{s}\left(k_{N} R_{\mathrm{c}}\right)\right]^{-1} \int_{0}^{\pi} \Psi_{N}\left(R_{\mathrm{c}}, \theta\right) \sin (s \theta) \mathrm{d} \theta .
$$

In Eq. (6) the number of basis functions is limited to $L^{\prime \prime}=k_{N} r_{\max }^{\mathrm{no}-\mathrm{RS}}$, where $r_{\max }^{\mathrm{no}-\mathrm{RS}}=21.4 \mathrm{~cm}$ is the maximum radius of the no-RS cavity. Circular waves with angular momentum $s>L$, which correspond to evanescent waves, are neglected.

Using the method of the "trial wave function" we were able to reconstruct 190 experimental wave functions of the rough no-RS half-circular billiard with the level number $N$ between 6 and 248 and 27 wave functions with $N$ between 250 and 435 [3]. Wave functions with $N \leq 35$ lie in the regime of Breit-Wigner ergodicity while the ones with $N>35$ belong to the regime of Shnirelman ergodicity. In the case of the RS billiard we reconstructed 31 wave functions with $N$ between 17 and 204 lying in the regime of Breit-Wigner ergodicity. As the quantitative measure of the sign assignment quality we chose the integral $\gamma \int_{\Gamma}\left|\Psi_{N}(r, \theta)\right|^{2} \mathrm{~d} l$ calculated along the billiard's rough boundary $\Gamma$, where $\gamma$ is the length of $\Gamma$. The remaining wave functions from the ranges $N=6-435$ and $N=17-204$ of the no-RS and RS billiards, respectively, were not reconstructed because of an accidental near-degeneration of the neighboring states or due to problems with the measurements 


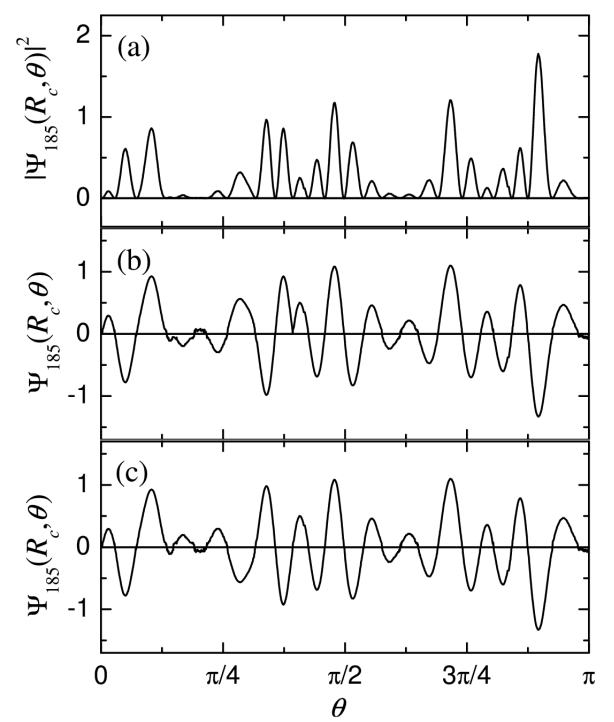

Fig. 3. (a) Squared wave function $\left|\Psi_{185}\left(R_{\mathrm{c}}, \theta\right)\right|^{2}$ (in arbitrary units) measured on a half-circle with radius $R_{\mathrm{c}}=19.25 \mathrm{~cm}$. (b) and (c) The "trial wave functions" $\Psi_{185}\left(R_{\mathrm{c}}, \theta\right)$ (in arbitrary units) with incorrectly and correctly assigned signs, respectively, which were used in the reconstruction of the wave functions $\Psi_{185}(r, \theta)$ of the billiard (see Fig. 4).

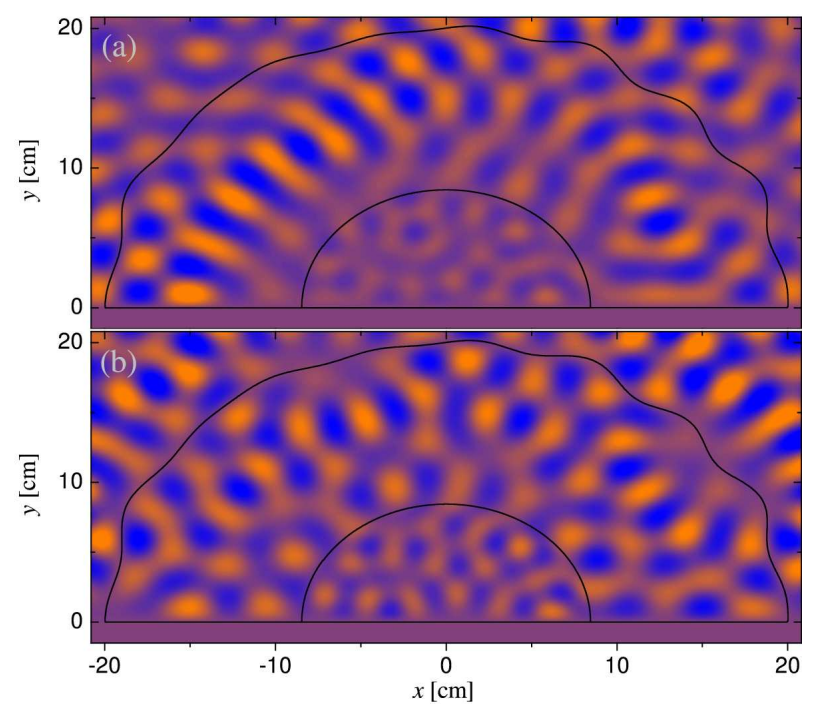

Fig. 4. (a) and (b) The wave functions $\Psi_{185}(r, \theta)$ of the chaotic microwave half-circular $\mathrm{RS}$ rough billiard reconstructed from the incorrect and correct "trial wave functions" shown in Fig. 3b and c, respectively. The amplitudes have been converted into a color scale with orange corresponding to large positive and blue corresponding to large negative values, respectively. Dimensions of the billiard are given in $\mathrm{cm}$. 
of $\left|\Psi_{N}\left(R_{\mathrm{c}}, \theta\right)\right|^{2}$ along a half-circle coinciding for its significant part with one of the nodal lines of $\Psi_{N}(r, \theta)$.

In Fig. 3a we show the squared wave function $\left|\Psi_{N}\left(R_{\mathrm{c}}, \theta\right)\right|^{2}, N=185$, of the RS billiard measured on the half-circle of radius $R_{\mathrm{c}}=19.25 \mathrm{~cm}$. The examples of the "trial wave functions" $\Psi_{185}\left(R_{\mathrm{c}}, \theta\right)$ of the RS billiard with not correctly assigned signs and correctly assigned signs are shown in Fig. 3b and c, respectively. The "trial wave function" presented in Fig. 3b was used in the reconstruction of the wave function $\Psi_{185}(r, \theta)$ shown in Fig. 4a, which evidently does not fulfill the boundary condition $\Psi_{185}\left(r_{\Gamma}, \theta_{\Gamma}\right) \simeq 0$. In Fig. $4 \mathrm{~b}$ we show properly reconstructed wave function $\Psi_{185}(r, \theta)$ of the RS billiard, which was obtained from the "trial wave function" presented in Fig. 3c. In this case the boundary condition $\Psi_{185}\left(r_{\Gamma}, \theta_{\Gamma}\right) \simeq 0$ is much better fulfilled.

\section{Structure of energy surface}

The properties of the measured wave functions may be further investigated by finding the structures of their energy surfaces [15]. The structure of the energy surface plays an important role in the identification whether a wave function exhibits a localized or ergodic behavior. To find the energy structure of the wave function $N$ of the RS billiard we extracted wave function amplitudes $C_{n l}^{(N)}=\langle n, l \mid N\rangle$ in the basis $n, l$ of a half-circular RS billiard (desymmetrized annular RS billiard) [25] with radius $r_{\max }^{\mathrm{RS}}$ and a half-circular Teflon insert of radius $R_{\mathrm{d}}$, where $n=1,2,3 \ldots$ enumerates the zeros of the radial function of the billiard and $l=1,2,3 \ldots$ is the angular quantum number. The moduli of amplitudes $\left|C_{n l}^{(N)}\right|$ and their projections into the energy surface for the experimental wave functions $N=22$ and $N=185$ of the RS billiard are shown in Fig. 5. The wave function $N=22$, which lies close to the low border of the Breit-Wigner regime $\left(N_{\mathrm{e}}=5\right)$, is quite well localized around the center of localization $n=3, l=5$. It is worth pointing out that the borders of localization and ergodicity in the rough billiards are not sharp. Therefore, it is possible to observe localized wave functions even for $N$ slightly higher than $N_{\mathrm{e}}$, i.e. in the Breit-Wigner regime. The wave function $N=185$, which lies nearby the border of the Shnirelman regime $\left(N_{\mathrm{W}}=208\right)$, is extended over the whole energy surface [13]. The full lines on the projection planes in Fig. 5a and b mark the energy surface of a half-circular annular ray-splitting billiard $H(n, l) \simeq E_{N}=k_{N}^{2}$ estimated from the formula $\left|H(n, l)-E_{N}\right| / E_{N} \leq 0.12$. The peaks $\left|C_{n l}^{(185)}\right|$ are spread almost perfectly along the line marking the energy surface.

The structures of the energy surfaces of the wave functions $N=30$ from the Breit-Wigner regime $(1<N \leq 35)$ and $N=413$ from the Shnirelman regime of the no-RS billiard are shown in Fig. 6 a and b, respectively. As expected, the energy structure of the wave function $N=30$ is similar to the energy structure of the wave function $N=185$ of the RS billiard. In the case of the regime of the 
(a)

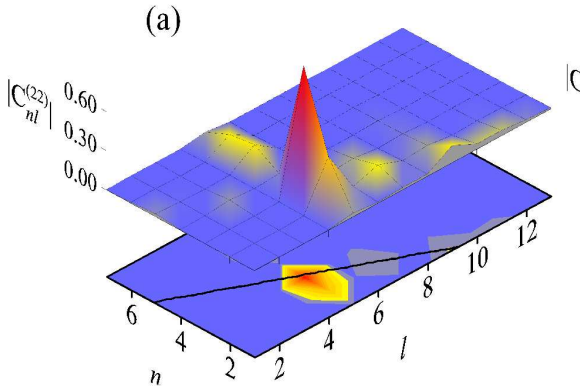

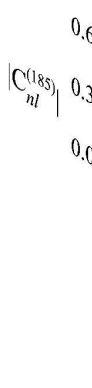

(b)

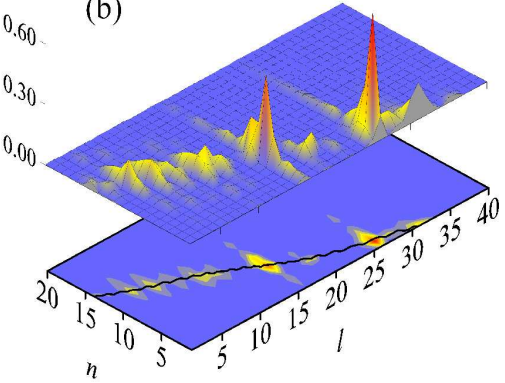

Fig. 5. Structure of the energy surface in the regime of Breit-Wigner ergodicity for the RS billiard. Here we show the moduli of amplitudes $\left|C_{n l}^{(N)}\right|$ for the wave functions: (a) $N=22, \nu_{22} \simeq 3.25 \mathrm{GHz}$ and (b) $N=185, \nu_{185} \simeq 8.77 \mathrm{GHz}$. The low wave function $N=22$ is localized, while the higher wave function $N=185$ is delocalized in the $n, l$ basis. Full lines show the semiclassical estimation of the energy surface (see the text).
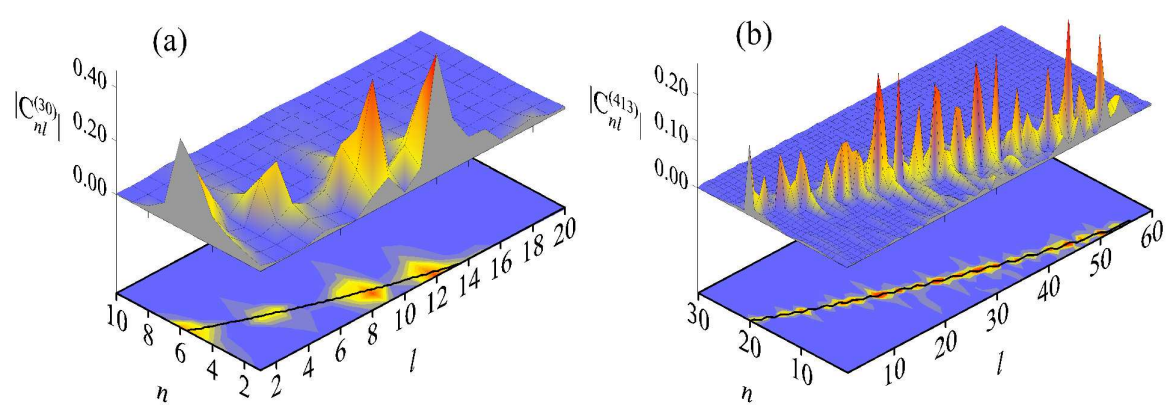

Fig. 6. Structure of the energy surface in the regime of Breit-Wigner and Shnirelman ergodicity for the no-RS billiard. We show the moduli of amplitudes $\left|C_{n l}^{(N)}\right|$ for the wave functions: (a) $N=30, \nu_{30} \simeq 4.09 \mathrm{GHz}$ and (b) $N=413, \nu_{413} \simeq 14.09 \mathrm{GHz}$. Both wave functions are delocalized in the $n, l$ basis. Full lines show the semiclassical estimation of the energy surface (see the text).

Shnirelman ergodicity the wave function $N=413$ is homogeneously distributed over the whole energy surface.

\section{Amplitude distribution}

Ergodic behavior of the wave functions of the billiards can be additionally tested by the evaluation of the amplitude distribution $P\left(\Psi_{N}\right)[26,27]$. For chaotic states the probability of finding the value $\Psi_{N}$ at any point inside the billiard should be distributed as a Gaussian, $P\left(\Psi_{N}\right) \sim \exp \left(-\beta \Psi_{N}^{2}\right)$. In Fig. 7a we show the amplitude distribution $P\left(\Psi_{N} A^{1 / 2}\right)$ of the wave function $N=22$ while in Fig. $7 \mathrm{~b}$ the distribution $P\left(\Psi_{N} A^{1 / 2}\right)$ of the wave function $N=185$ of the RS billiard is presented. The width of the amplitude distributions $P\left(\Psi_{N}\right)$ was rescaled to unity by multiplying normalized to unity wave functions by the factor $A^{1 / 2}$, where $A$ de- 

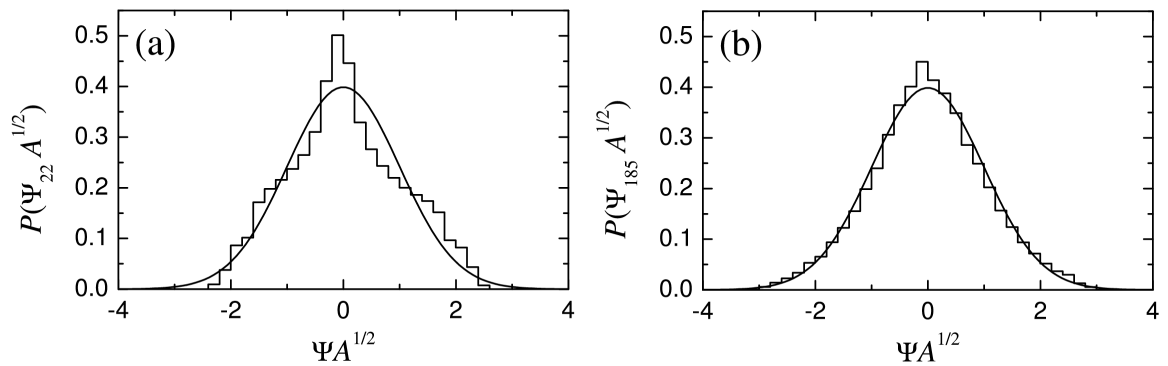

Fig. 7. The amplitude distribution $P\left(\Psi_{N}^{\text {in }} A^{1 / 2}\right)$ of the wave functions $N=22$ (a) and $N=185$ (b) from the regime of the Breit-Wigner ergodicity of the RS billiard. The amplitude distributions were constructed as histograms with bin equal to 0.2 . The width of the distribution $P(\Psi)$ was rescaled to unity by multiplying normalized to unity wave function by the factor $A^{1 / 2}$, where $A$ denotes billiard's area. Full line shows standard normalized Gaussian prediction $P_{0}\left(\Psi A^{1 / 2}\right)=(1 / \sqrt{2 \pi}) \exp \left(-\Psi^{2} A / 2\right)$.

notes billiard's area (see formula (23) in [27]). The distributions were constructed as normalized to unity histograms with the bin equal to 0.2. Because the wave function $N=22$ lies close to the localization border its amplitude distribution $P\left(\Psi_{N} A^{1 / 2}\right)$ departures significantly from the Gaussian prediction (see Fig. 7a). For the higher wave function $N=185$ lying also in the regime of Breit-Wigner ergodicity, but being close to the border of the Shnirelman ergodicity, the distribution of $P\left(\Psi_{N} A^{1 / 2}\right)$ is in good agreement with the standard normalized Gaussian prediction $P_{0}\left(\Psi A^{1 / 2}\right)=(1 / \sqrt{2 \pi}) \exp \left(-\Psi^{2} A / 2\right)$.

The amplitude distribution $P\left(\Psi_{N}\right)$ of the wave functions $N=30$ and $N=$ 413 of the no-RS billiard lying in the regimes of Breit-Wigner and Shnirelman ergodicity, respectively, are shown in Fig. 8a and b. We checked that for all wave
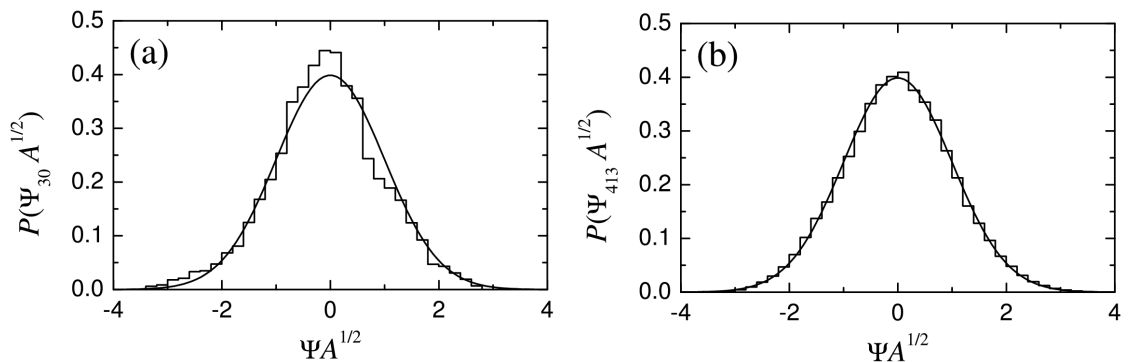

Fig. 8. The amplitude distribution $P\left(\Psi_{N}^{\text {in }} A^{1 / 2}\right)$ of the wave functions $N=30$ and $N=$ 413 of the no-RS billiard from the regimes of the Breit-Wigner (a) and the Shnirelman ergodicity (b), respectively. The amplitude distributions were constructed as histograms with bin equal to 0.2 . The width of the distribution $P(\Psi)$ was rescaled to unity by multiplying normalized to unity wave function by the factor $A^{1 / 2}$, where $A$ denotes billiard's area. Full line shows standard normalized Gaussian prediction $P_{0}\left(\Psi A^{1 / 2}\right)=$ $(1 / \sqrt{2 \pi}) \exp \left(-\Psi^{2} A / 2\right)$. 
functions of the no-RS billiard lying in the regime of Shnirelman ergodicity the distribution of $P\left(\Psi_{N} A^{1 / 2}\right)$ is in good agreement with the standard normalized Gaussian prediction, which is not always true for wave functions from the BreitWigner regime (see Fig. 8a).

\section{Number of nodal domains}

The number of nodal domains $\aleph_{N}$ vs. the level number $N$ in the chaotic microwave RS rough billiard is plotted in Fig. 9. All included wave functions $N=17-204$ lie in the regime of Breit-Wigner ergodicity. The full line in Fig. 9 shows the least squares fit $\aleph_{N}=a_{1} N+b_{1} \sqrt{N}$ of the experimental data, where $a_{1}=0.055 \pm 0.017, b_{1}=1.006 \pm 0.209$. The coefficient $a_{1}=0.055 \pm 0.017$ is smaller than the prediction of the percolation model of Bogomolny and Schmit [2], $\aleph_{N} / N \simeq 0.062$, though it coincides with it within the error limits. The errors of the coefficients $a_{1}$ and $b_{1}$ are relatively high because the number of nodal domains fluctuates significantly in the function of the level number $N$, which was also demonstrated in [1] (see Fig. 5).

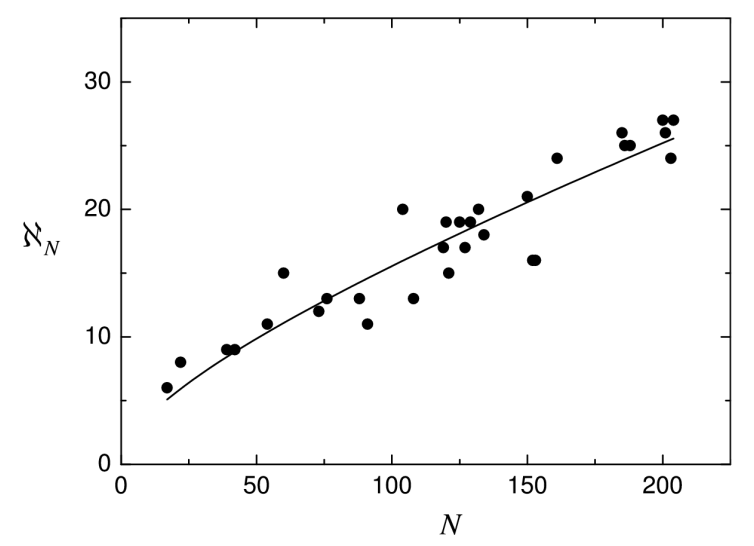

Fig. 9. The number of nodal domains $\aleph_{N}$ (full circles) versus a level number $N$ for the regime of Breit-Wigner ergodicity of the RS billiard. Full line shows the least squares fit $\aleph_{N}=a_{1} N+b_{1} \sqrt{N}$ to the experimental data (see the text), where $a_{1}=0.055 \pm$ $0.017, b_{1}=1.006 \pm 0.209$. The prediction of the theory of Bogomolny and Schmit [2], $a_{1}=0.062$.

The number of nodal domains $\aleph_{N}$ vs. the level number $N$ in the chaotic microwave no-RS rough billiard is plotted in Fig. 10. Here we used wave functions $N=6-435$ of the no-RS billiard. The full line in Fig. 10 shows the least squares fit $\aleph_{N}=a_{2} N+b_{2} \sqrt{N}$ of the experimental data, where $a_{2}=0.058 \pm 0.006, b_{2}=1.075 \pm$ 0.088 , calculated for higher levels $80 \leq N \leq 435$. The coefficient $a_{2}=0.058 \pm 0.006$ evaluated in the regime of Shnirelman ergodicity is closer to the prediction of the percolation model than the one found for the regime of Breit-Wigner ergodicity. 


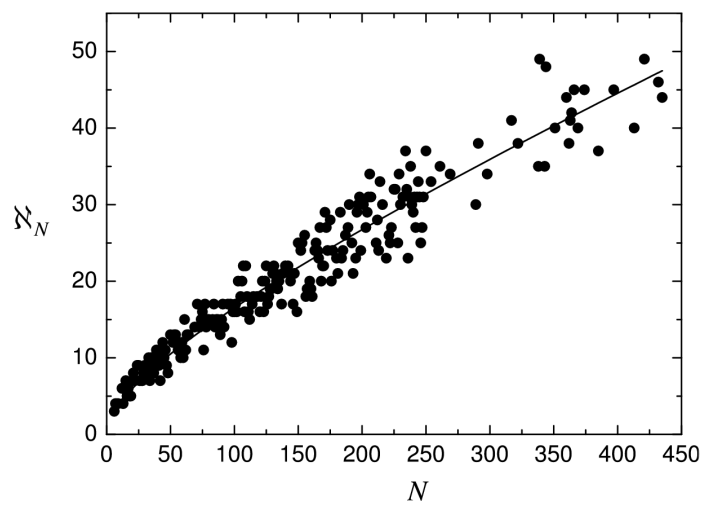

Fig. 10. The number of nodal domains $\aleph_{N}$ (full circles) versus a level number $N$ for the regime of Shnirelman ergodicity of the no-RS billiard. Full line shows the least squares fit $\aleph_{N}=a_{1} N+b_{1} \sqrt{N}$ to the experimental data (see the text), where $a_{1}=0.058 \pm$ $0.006, b_{1}=1.075 \pm 0.088$. The prediction of the theory of Bogomolny and Schmit [2], $a_{1}=0.062$.

The second term in the least squares fits corresponds to a contribution of boundary domains, i.e. domains, which include the billiard boundary. Numerical calculations of Blum et al. [1] performed for the Sinai and stadium billiards showed that the number of boundary domains scales as the number of the boundary intersections, that is as $\sqrt{N}$. Present results clearly suggest that in the rough billiards in the regimes of Breit-Wigner and Shnirelman ergodicity the boundary domains also significantly influence the scaling of the number of nodal domains $\aleph_{N}$, leading to the departure from the predicted scaling $\aleph_{N} \sim N$.

\section{Signed area distribution}

Reconstructed wave functions of the RS and no-RS billiards may be also used for the calculations of the signed area distribution $\Sigma_{A}$ introduced by Blum et al. [1]. The signed area distribution is defined as follows: $\Sigma_{A}=\left\langle\left(A_{+}-A_{-}\right)^{2}\right\rangle / A^{2}$, where $A_{ \pm}$is the total area where the wave function is positive (negative) and $A$ is the billiard area. It is predicted [1] that for chaotic wave functions the signed area distribution should converge in the asymptotic limit to $\Sigma_{A} \simeq 0.0386 N^{-1}$. It is worth noting that for integrable billiards $\Sigma_{A}$ displays nonuniversal behavior. For example, for a rectangular billiard of size $L_{a} \times L_{b}$, $\Psi_{n, m}(x, y) \propto \sin \left(n x \pi / L_{a}\right) \sin \left(m y \pi / L_{b}\right), \quad \Sigma_{A}=0$ if $n$ or $m$ are even. If $n$ and $m$ are odd $\Sigma_{A}=1 /(\mathrm{nm})^{2}$. For a circular billiard for all doublet states (angular momentum $\ell \neq 0) \Sigma_{A}=0$.

Figure 11a shows the normalized signed area distribution $N \Sigma_{A}$ for the microwave RS rough billiard. The points in Fig. 11a were obtained by averaging over 7 consecutive eigenstates. For low level numbers $N<208$ lying in the regime of Breit-Wigner ergodicity the normalized distribution $N \Sigma_{A}$ is scattered around the 
predicted asymptotic limit. A slow convergence of $N \Sigma_{A}$ at low level numbers $N$ was also observed for the Sinai and stadium billiards [1]. In the case of the Sinai billiard this phenomenon was attributed to the presence of corners with sharp angles, which are also present in the microwave rough RS billiard. According to Blum et al. [1] the effect of corners on the wave functions is accentuated at low energies.
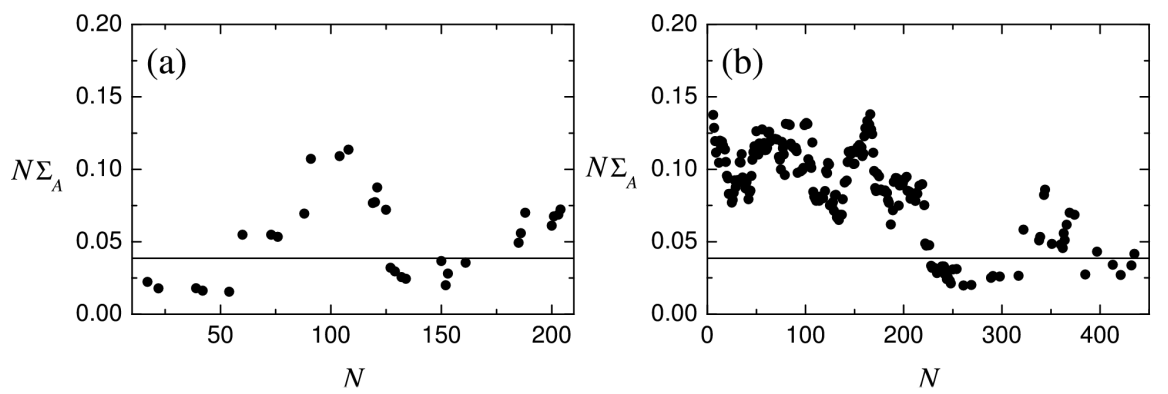

Fig. 11. The normalized signed area distribution $N \Sigma_{A}$ for the chaotic microwave half-circular rough billiards. The distribution $N \Sigma_{A}$ for the RS billiard (a) and for the no-RS billiard (b). Full line shows predicted by the theory asymptotic limit $N \Sigma_{A} \simeq 0.0386$, Blum [1].

In Fig. 11b the normalized signed area distribution $N \Sigma_{A}$ is shown for the microwave no-RS rough billiard. For lower states $6 \leq N \leq 250$ the points in Fig. $11 \mathrm{~b}$ were obtained by averaging over 20 consecutive eigenstates while for higher states $N>250$ the averaging over 5 consecutive eigenstates was applied. For level numbers $N<220$ the normalized distribution $N \Sigma_{A}$ is above the predicted asymptotic limit, however, for $220<N \leq 435$ it more closely approaches the asymptotic limit. This provides a strong evidence that the signed area distribution $\Sigma_{A}$ can be used as a useful criterion of quantum chaos.

\section{Conclusions}

We measured the wave functions of the chaotic rough microwave billiards with and without ray-splitting properties. The wave functions measured in the RS billiard laid in the regime of Breit-Wigner ergodicity. The wave functions measured in the no-RS billiard belonged mainly to the regime of Shnirelman ergodicity. Following the results of percolation-like model proposed by [2] we confirmed that in the limit $N \rightarrow \infty$ the least squares fit of the experimental data obtained for the regime of Shnirelman ergodicity in the no-RS billiard yields the asymptotic number of nodal domains $\aleph_{N} / N \simeq 0.058 \pm 0.006$ that is close to the theoretical prediction $\aleph_{N} / N \simeq 0.062$ [2]. In the case of the regime of Breit-Wigner ergodicity in the RS billiard $(17 \leq N \leq 204)$ we obtained in the asymptotic limit $\aleph_{N} / N \simeq 0.055 \pm 0.017$. This value is smaller than the one obtained for the no-RS 
billiard, though it coincides with the theoretical prediction within the error limits. Finally, we demonstrate that the signed area distribution $\Sigma_{A}$ approaches for high level number $N$ of the no-RS billiard theoretically predicted asymptotic limit $0.0386 N^{-1}[1]$. The experimental results presented in this paper suggest that some properties of nodal domains of wave functions of chaotic RS billiards, such as the asymptotic number of nodal domains $\aleph_{N} / N$, are the same like in no-RS billiards.

\section{Acknowledgments}

This work was supported by Ministry of Science and Information Society Technologies grant No. 2 P03B 04724.

\section{References}

[1] G. Blum, S. Gnutzmann, U. Smilansky, Phys. Rev. Lett. 88, 114101 (2002).

[2] E. Bogomolny, C. Schmit, Phys. Rev. Lett. 88, 114102 (2002).

[3] N. Savytskyy, O. Hul, L. Sirko, Phys. Rev. E 70, 056209 (2004).

[4] R. Blümel, T.M. Antonsen, B. Georgeot, E. Ott, R.E. Prange, Phys. Rev. Lett. 76, 2476 (1996); Phys. Rev. E 53, 3284 (1996).

[5] L. Sirko, P.M. Koch, R. Blümel, Phys. Rev. Lett. 78, 2940 (1997).

[6] Sz. Bauch, A. Błędowski, L. Sirko, P.M. Koch, R. Blümel, Phys. Rev. E 57, 304 (1998).

[7] R. Blümel, P.M. Koch, L. Sirko, Found. Phys. 31, 269 (2001).

[8] Y. Hlushchuk, A. Kohler, Sz. Bauch, L. Sirko, R. Blümel, M. Barth, H.-J. Stöckmann, Phys. Rev. E 61, 366 (2000).

[9] N. Savytskyy, A. Kohler, Sz. Bauch, R. Blümel, L. Sirko, Phys. Rev. E 64, 036211 (2001).

[10] R. Schäfer, U. Kuhl, M. Barth, H.-J. Stöckmann, Found. Phys. 31, 475 (2001).

[11] N. Savytskyy, L. Sirko, Phys. Rev. E 65, 066202 (2002).

[12] H.-J. Stöckmann, Quantum Chaos, an Introduction, Cambridge University Press, Cambridge 1999.

[13] Y. Hlushchuk, A. Błędowski, N. Savytskyy, L. Sirko, Phys. Scr. 64, 192 (2001).

[14] Y. Hlushchuk, L. Sirko, U. Kuhl, M. Barth, H.-J. Stöckmann, Phys. Rev. E 63, 046208 (2001).

[15] K.M. Frahm, D.L. Shepelyansky, Phys. Rev. Lett. 78, 1440 (1997).

[16] L. Sirko, Sz. Bauch, Y. Hlushchuk, P.M. Koch, R. Blümel, M. Barth, U. Kuhl, H.-J. Stöckmann, Phys. Lett. A 266, 331 (2000).

[17] K.M. Frahm, D.L. Shepelyansky, Phys. Rev. Lett. 79, 1833 (1997).

[18] A. Shnirelman, Usp. Mat. Nauk. 29, N6, 18 (1974).

[19] L.C. Maier, J.C. Slater, J. Appl. Phys. 23, 68 (1952).

[20] S. Sridhar, Phys. Rev. Lett. 67, 785 (1991).

[21] D.H. Wu, J.S.A. Bridgewater, A. Gokirmak, S.M. Anlage, Phys. Rev. Lett. 81, 2890 (1998). 
[22] C. Dembowski, H.-D. Gräf, A. Heine, R. Hofferbert, H. Rehfeld, A. Richter, Phys. Rev. Lett. 84, 867 (2000).

[23] J. Stein, H.-J. Stöckmann, U. Stoffregen, Phys. Rev. Lett. 75, 53 (1995).

[24] M. Hentschel, K. Richter, Phys. Rev. E 66, 056207 (2002).

[25] A. Kohler, R. Blümel, Phys. Lett. A 238, 271 (1998).

[26] M.V. Berry, J. Phys. A 10, 2083 (1977).

[27] S.W. McDonald, A.N. Kaufman, Phys. Rev. A 37, 3067 (1988). 